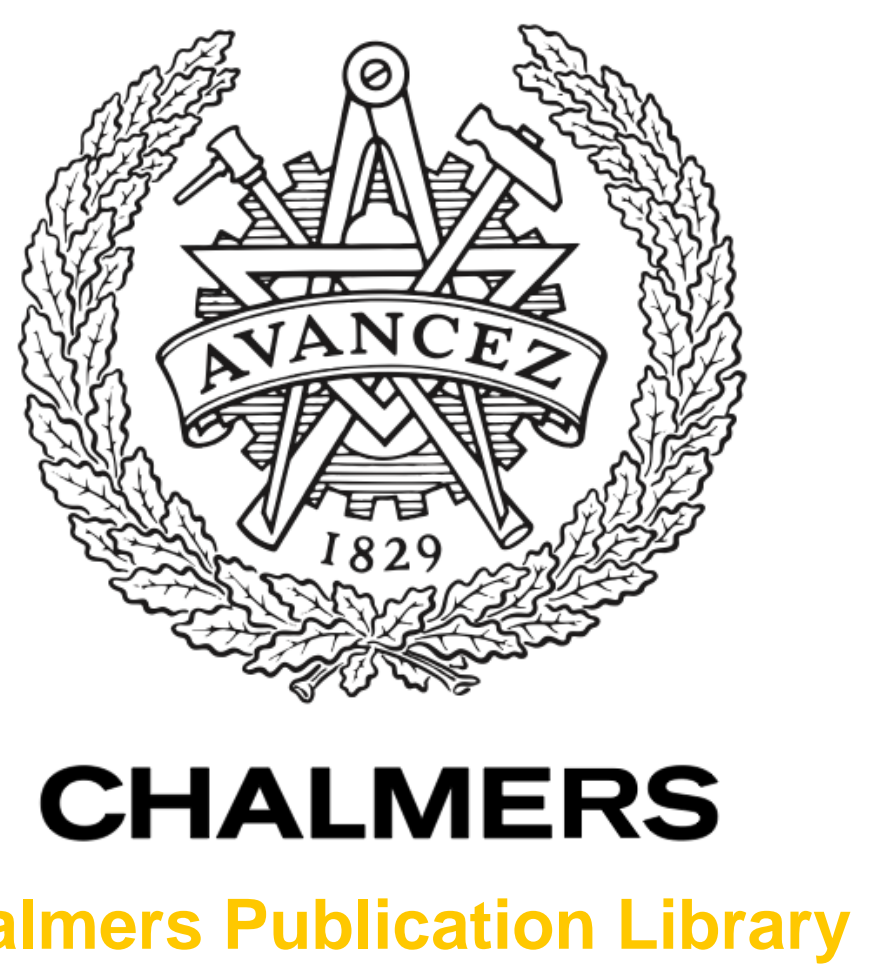

\title{
Optimal parameters of monolithic high-contrast grating mirrors
}

This document has been downloaded from Chalmers Publication Library (CPL). It is the author's version of a work that was accepted for publication in:

\section{Optics Letters (ISSN: 0146-9592)}

Citation for the published paper:

Marciniak, M. ; Gbski, M. ; Dems, M. et al. (2016) "Optimal parameters of monolithic highcontrast grating mirrors". Optics Letters, vol. 41(15), pp. 3495-3498.

http://dx.doi.org/10.1364/OL.41.003495

Downloaded from: http://publications.lib.chalmers.se/publication/239527

Notice: Changes introduced as a result of publishing processes such as copy-editing and formatting may not be reflected in this document. For a definitive version of this work, please refer to the published source. Please note that access to the published version might require a subscription. 


\title{
Optimal Parameters of Monolithic High Contrast Grating Mirrors
}

\author{
Magdalena Marciniak ${ }^{1}$, Marcin Gębski $^{1,2}$, Maciej Dems $^{1}$, ERIK Haglund ${ }^{3}$, Anders Larsson ${ }^{3}$, \\ MAJID RIAZIAT ${ }^{4}$, JAMES A. LOTT ${ }^{2}$, AND TOMASZ CZYSZANOWSKI ${ }^{1,}$ \\ ${ }^{1}$ Institute of Physics, Lodz University of Technology, ul. Wolczanska 219, 90-924 Lodz, Poland \\ ${ }^{2}$ Institute of Solid State Physics and Center of Nanophotonics, Technische Universität Berlin, Hardenbergstraße 36, D-10623 Berlin, Germany \\ ${ }^{3}$ Photonics Laboratory, Department of Microtechnology and Nanoscience, Chalmers University of Technology, SE-41296 Göteborg, Sweden \\ ${ }^{4}$ OEpic Semiconductors Inc., 1231 Bordeaux Drive, Sunnyvale, California, 94089, United States of America \\ *Corresponding author: tomasz.czyszanowski@p.lodz.pl

In this paper a fully vectorial numerical model is used to search for the construction parameters of monolithic high contrast grating (MHCG) mirrors providing maximal power reflectance. We determine the design parameters of highly reflecting MHCG mirrors where the etching depth of the stripes is less than two wavelengths in free space. We analyze MHCGs in a broad range of real refractive index values corresponding to most of all of the common optoelectronic materials in use today. Our results comprise a complete image of possible highly reflecting MHCG mirror constructions for potential use in optoelectronic devices and systems. We support the numerical analysis by experimental verification of the high reflectance via a GaAs MHCG designed for a wavelength of $980 \mathrm{~nm}$. ๑ 2016 Optical Society of America

OCIS codes: (050.1950) Difraction gratings; (050.6624) Subwavelenght structures; (140.7260) Vertical cavity surface emitting lasers.

http://dx.doi.org/10.1364/ao.XX.XXXXXX

Mirrors of very high power reflectance play important roles in numerous applications in optoelectronics, for example in waveguides [1, 2], resonant-cavity photodetectors [3], laser diodes, and more. However, one of the most prominent examples of the use of highly reflecting mirrors in optoelectronics is their use in Vertical Cavity Surface Emitting Lasers (VCSELs) where the mirrors must provide power reflectance values typically higher than $0.99(99 \%)$ [4]. In VCSELs a very short cavity length that is equal to an integer multiple of a half-lambda optical thickness $(\lambda / 2, \lambda, 3 \lambda / 2$, etc. $)$ is used which typically allows single longitudinal mode operation. The short cavity and the associated very thin quantum well active region layers have the characteristic that the optical gain per round-trip pass through the cavity is far less than that for typical edge-emitting constructions of in-plane semiconductor lasers. To compensate for the VCSEL's short cavity the power reflectance of the mirrors, which are typically in the form of distributed Bragg reflectors (DBRs), must be very high at the lasing wavelength. High power reflectance can be achieved for example by using stack of 20 pairs or more of quarter-wavelength-thick layers of $\mathrm{GaAs}$ and $\mathrm{Al}_{0.9} \mathrm{Ga}_{0.1} \mathrm{As}$ which is a typically used DBR construction in arsenide-based VCSELs emitting at a peak wavelength of about $980 \mathrm{~nm}[5,6]$. The requirement of the combination of a short cavity and high power reflectance DBR mirrors leads to a bizarre construction where the VCSEL's cavity thickness can be less than $2 \%$ of the total combined thickness of the two DBRs.

High refractive index contrast grating (HCG) mirrors are one very appealing answer to address the issue of VCSEL miniaturisation $[7,8]$. These kinds of mirrors can be only about a halfwavelength thick, and they offer a reflection stopband that is twice as broad as that for conventional all-semiconductor DBR mirrors. Typical HCG layers consist of high refractive index stripes surrounded on all sides by low-refractive-index media. Since the HCG is the top layer of the laser, air is on one side of the mirror and on the other side a low refractive index material must serve as part of the laser's optical cavity [9-11].

The low refractive index materials are dielectrics (air or oxides) which are electric insulators with low thermal conductivities that typically deteriorate the temperature performance and thus the efficiency of their given VCSELs. To overcome these limitations, Goeman et al. [12] proposed "giant reflectivity to order grating" (GIRO-grating) undoped GaAs and InP monolithic grating mirrors. The power reflectance $(R)$ of the InP GIRO-grating reflector was measured to b e 0.7 at $1480 \mathrm{~nm}$ to just above 0.85 at $1560 \mathrm{~nm}$ (both $\pm 5 \%$ ) for TM-polarized light at normal incidence. A similar measured result for TM-polarized light with $R \sim 0.8$ at $1550 \mathrm{~nm}$ for an undoped GaAs GIRO-grating reflector was demonstrated. Furthermore, Lee et al. [13] reported a GaN monolithic "surface grating reflector" with $R \sim 0.92$ at $442 \mathrm{~nm}$ and $R \sim 0.8$ at $532 \mathrm{~nm}$ for TE-polarized light. There are other interesting reflector designs with wide high reflectance bandwidths including the hybrid grating [14] where a passive or active III-V layer is followed by a grating of lower index such as $\mathrm{Si}$, and the zero contrast grating [15] which consists of a grating material layer (surrounded by a low index material such as air with an underlying layer of the exact same material followed by a different substrate material). As we show in [16], these vari- 
ous forms of monolithic high contrast grating (MHCG) mirrors: 1) can be as thin as a quarter wavelength; 2) can be etched in any number of arbitrary materials that are transparent and that possess a refractive index that is larger than 1.75; and 3) may have a high-power-reflectance stopband that is comparable to the stopband of typical GaAs/AlGaAs DBRs.

In [16] we considered MHCG with minimal etching depths of MHCG stripes. However, there are a plethora of other designs with larger etching depths that result in MHCG reflectors with very high power reflectance for a given design wavelength and over a given stopband. In this letter we perform a more detailed numerical analysis of the power reflectance characteristics of stand-alone MHCG mirrors. We validate our numerical model by comparing simulated and experimental results for an (u)GaAs $980 \mathrm{~nm}$ MHCG reflector. We also perform an exhaustive numerical analysis of the geometrical parameters of MHCGs composed of materials with real refractive indices from 1.75 to 4 which covers most all of the common optoelectronic materials in use today. In the analysis we consider TE polarisation only (electric field parallel to the MHCG stripes) since a pre-existing analysis of TM polarisation showed narrower stopbands and larger sensitivity to possible imperfections of MHCGs with respect to TE configurations due to the high intensity of the optical field at the edges of MHCGs.

A schematic structure of an MHCG mirror, which we consider in our analysis, is shown in Fig.1a. To determine the power reflectance of an MHCG mirror we use the Plane-Wave Reflection Transformation (PWRT) method [17]. In calculations we consider a single period of an MHCG combined with periodic boundary conditions. To reach convergence 60 plane-waves were used for a single MHCG period. We calculate the power reflectance as the ratio of the intensity of the zeroth diffraction order wave reflected from the mirror to the intensity of a planewave that is normally-incident on the mirror from below (see the arrow in Fig.1a). Although our assumption of an infinite surface grating structure and plane-wave reflections may seem unrealistic, we find that our results are very close to the case of modelling a finite grating mirror that is impinged by incident waves with a Gaussian-shaped optical intensity profile since the fundamental-mode Gaussian profile is d ominant in both cases [16]. We perform our analysis of the MHCG's power reflectance as a function of the MHCG's geometrical parameters, namely: the MHCG period $L$; the MHCG stripe width $a$, and the stripe height $h$ as shown in Fig.1a. In the analysis we use the fill factor of the MHCG stripes which we define as $F=a / L$. All of the spatial parameters are normalized with respect to the wavelength.

Fig.1b illustrates an example simulated map of the power reflectance $R$ for an MHCG as a function of the grating stripe period $L$ and height $h$ for a fixed value of the fill factor $F=0.4$ and with a constant refractive index $n=2$. The fill factor is chosen to maximize the number of high reflectance a reas (islands). However, the $R$ maxima in Fig.1b do not include all of the power reflectance maxima. The complete set of maxima may only be seen if we also vary the fill factor $F$ (whereas $F$ is constant in Fig.1b). The islands of high reflectance (defined here as the regions for which $R>0.5$ ) are comprised of multiple smaller maxima [18]. A closer inspection of those regions reveals what we call local maxima of power reflectance (LMPR), where the peak of $R$ values within a given LMPR are very close to unity. These LMPRs are however, located in planes of different fill factors. Hence one must perform a three-dimensional optimization with respect to the maximal value of power reflectance

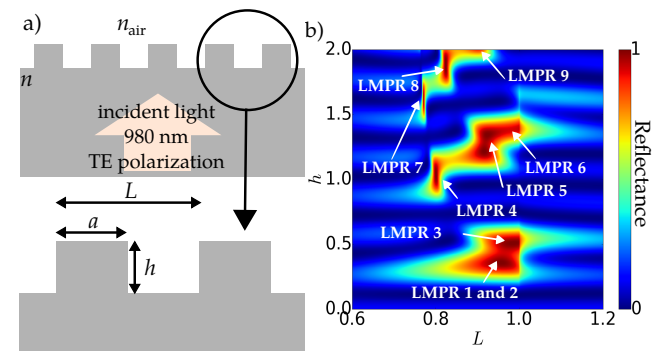

Fig. 1. a) Schematic structures of an MHCG reflector, where $L$ is the surface grating period of the MHCG, $a$ is the stripe width, $h$ is the height of the rectangular stripes, $n$ is the real refractive index of mirror material, and $n_{\text {air }}$ is the refractive index of the surrounding air. The direction of incident light is indicated by the wide upward arrow. b) The power reflectance map with reflectance $R$ as a function of $L$ and $h$ (normalized with the respect to the wavelength) for a fixed $F=0.4$ and $n$ $=2.0$ for the MHCG material. The local maxima of power reflectance (LMPR) are indicated.

considering $L, a$, and $h$ as variables for the given refractive index. In Fig.1b eight LMPRs are indicated by labeled arrows. Fig. 2 shows the optical field intensity distribution within single MHCG stripes for parameters which correspond to the given LMPRs for $n=2$ and $n=3.5$.

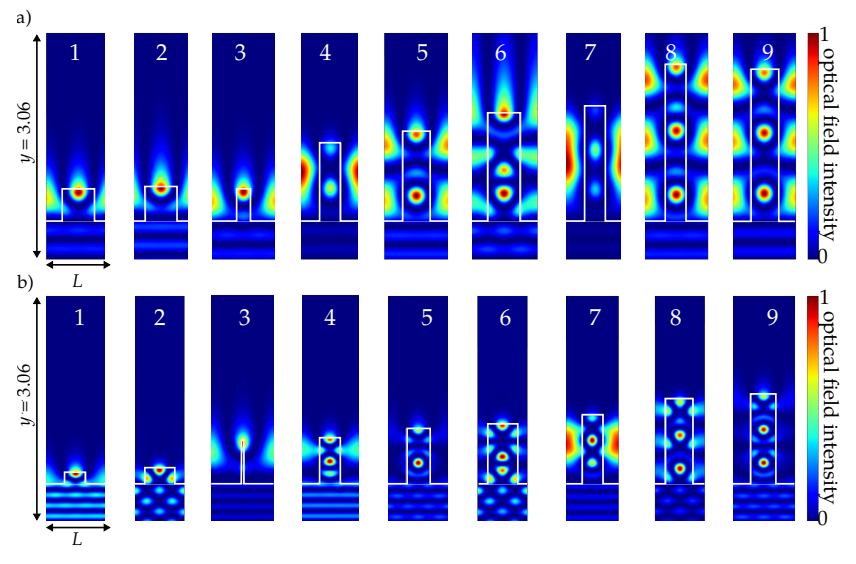

Fig. 2. Optical field intensity distribution in MHCG stripes for different constructions of the MHCG mirrors corresponding to different LMPRs for a refractive index equal to: a) 2.0; and b) 3.5. The numbers in each figure assign the numbers of LMPRs; the y at the horizontal axis is a normalized distance with respect to the wavelength. The exact parameters of the LMPRs can be found from Fig.3.

By carefully inspecting Fig.2 one can notice that the optical intensity distributions might be classified into three groups, which differ due to the number of dominant maxima found within the stripes. The distributions of the optical field intensity corresponding to: 1) LMPRs 1-3 contain a single dominant lobe; 2) LMPRs 4-6 contain two dominant lobes; and 3) LMPRs 7-9 contain three dominant lobes. Our classification is in line with the classification based on the power reflectance map in Fig.1b, which has three distinct LMPR groups located in each of the islands of high reflectance $(R>0.5)$. It is worth mentioning that in most of the cases we observe here, the maximal optical intensity is located within the grating stripes. The only exceptions 
are LMPRs 4 and 7. In these two cases the optical field intensity is dominantly confined between the stripes and only a small portion of the field resides in the stripes. This effect is more pronounced when the refractive index $n=2$ (as in Fig.2(a)), compared to when $n=3.5$ (as in Fig.2(b)).

In Fig. 3 we plot the parameters of the MHCGs corresponding to the LMPRs as a function of the refractive index for $n=1.75$ to 4 . To trace the modifications of MHCG parameters in a convenient manner the spatial dimensions $h, L$, and $a$ are multiplied by the refractive index $n$ of the MHCG. All the curves have been calculated with a 0.002 step of refractive index and each point has been found based on a three dimensional maximization of the power reflectance as a function of $L, a$ and $h$.
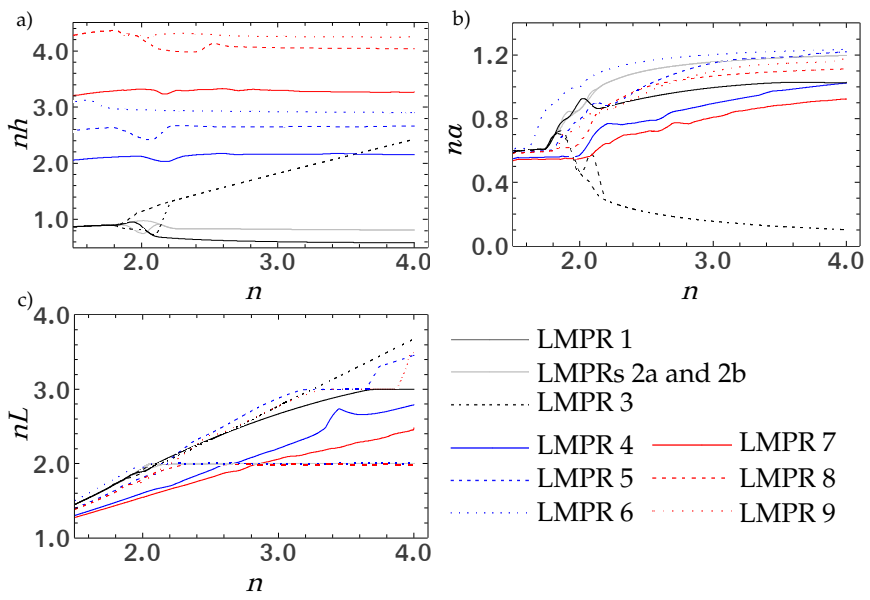

Fig. 3. a) Optical height of the stripes $n h$, b) optical length of the stripes $n a$ and c) optical length of the period $n L$ as functions of the refractive index $n$ for the given LMPRs. The geometrical parameters of MHCG mirrors are normalized with respect to the wavelength.

Fig.3(a) illustrates the impact of a modification of the etching depth as a function of the refractive index on the LMPRs. The general tendency is that the optical height of the MHCG stripes $(n h)$ that corresponds to most of the LMPRs is nearly constant, which indicates a proportionality between $h$ and $1 / n$. A more careful inspection reveals that in the case of lower refractive indices the analyzed functions are slowly increasing with $n$. This results from an increasing confinement of the optical field within the stripes with $n$ which is evident by comparing Figs.2a and $2 \mathrm{~b}$. In the range of low refractive indices $(n<1.9)$ one can distinguish six LMPRs. The LMPR corresponding to the smallest $h$ splits in five different LMPRs in the range $1.9<n<2.1$ which merge again into three separate LMPRs. Splitting and merging of the LMPRs can be observed for two other parameters, $a$ in Fig.3b and $L$ in Fig.3c. In the case of the LMPR of largest considered $h$ it splits into two LMPRs at $n>2$. A peculiar behaviour can be observed in the case of LMPR 3. It is the only one for which $n h$ increases linearly which turns into constant dependence of $h$ as the function of $n$. Fig.3b shows that the analysed LMPRs correspond to $n a \approx 0.55$ for $n<2$. Further increase of $n$ leads to the increase of $n a$ and spread of its values in the range from 0.8 to 1.2 . The only exceptions is LMPR 3 for which $n a$ reduces significantly although no significant modification of the optical field is observed. The MHCG periods corresponding to LMPRs are located within the dual mode region [18]. In the range of low refractive indices $n<2, L$ is close to the diffraction limit
(Fig.3c) for all LMPRs. Increase of the refractive index leads to incremental moving away LMPRs from diffraction limit. For $n>2.2$ one can observe that $n L$ becomes constant for LMPRs 2, 6 and 8. Similar is observed for LMPRs 5 and 9 for $n>3.2$.

We performed a spectral analysis in which we define the high reflectance stopband to be given as the ratio $\Delta \lambda / \lambda$ where $\Delta \lambda$ is the spectral range in which the power reflectance is greater than a particular assumed value of power reflectance (e.g. $>0.90$ or $>0.99$ ) and $\lambda$ is the wavelength corresponding to maximal power reflectance. Fig.4 illustrates maximal power reflectance together with 0.9 and 0.99 stopbands of all consecutive LMPRs. The actual results confirm the observations reported in [17] on minimal refractive index $(n=1.75)$ assuring the power reflectance above 0.99 .

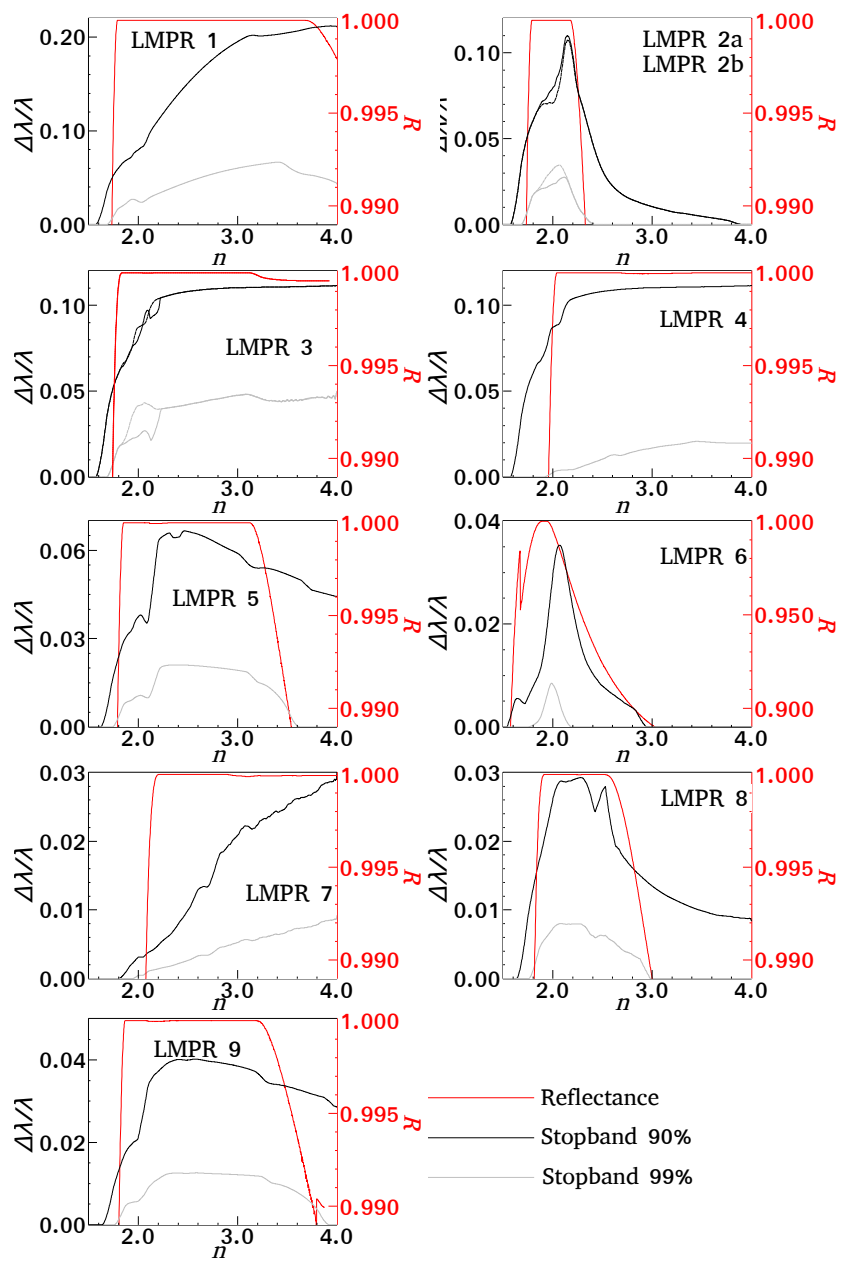

Fig. 4. Normalized power reflectance stopband $\Delta \lambda / \lambda$ calculated for different LMPRs for power reflectances $R=0.9$ (gray curve) and $R=0.99$ (black curve), and power reflectance $R$ as functions of the MHCG refractive index (red curve). The variation of the refractive index occurs together with modification of geometrical parameters of MHCGs according to Fig.3.

Comparing all results from Fig.4 and relating the numbers of LMPRs to their etching depths $h$ one can notice that stopbands increase monotonically with the increase of $h$ in the analysed range of refractive indices for only two LMPRs, namely for LMPRs 4 and 7. The results show that LMPR 1 is dominant over the rest of the LMPRs due to the size of the stopbands in the range of refractive indices $2.4<n<4.0$. For lower refractive 
indices LMPRs 2 and 3 reveal larger stopbands.

To support the numerical analysis we performed experimental verification of the high reflectance of an undoped GaAs MHCG surface grating designed for a peak TE-polarized $R$ at a wavelength of $980 \mathrm{~nm}$. The structure was designed according to LMPR 1 for $n=3.51$ and consecutive MHCG parameters are $L=818.3 \mathrm{~nm}, F=0.35$ and $h=164.5 \mathrm{~nm}$. A $4 \mathrm{~mm}$ by $4 \mathrm{~mm}$ area of MHCG was implemented by electron beam lithography (EBL) and etched using inductively coupled plasma reactive ion etching (ICP RIE). The scanning electron microscopy (SEM) image of the resulting MHCG is shown in Figs.5a and 5b.
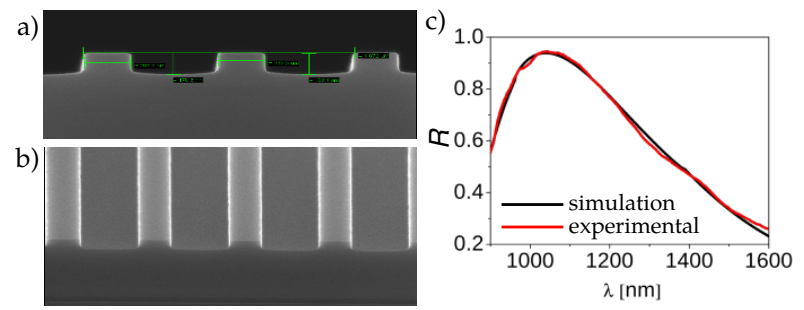

Fig. 5. a) Cross-section and b) top view SEM images of the fabricated undoped GaAs MHCG according to LMPR 1 for $n=3.51$ and $\lambda=980 \mathrm{~nm}$; and c) experimental and theoretical relative power reflectance spectra of the undoped GaAs MHCG in an undoped GaAs substrate of thickness $\sim 200 \mu \mathrm{m}$ with a quarter-lambda-thick SiN anti-reflection coating on the bulk GaAs surface opposite to the grating, for light polarized parallel to the grating stripes (TE polarization). The theoretical results are for incoming planewaves that are parallel to the surface.

The power reflectance m easurement s etup consists of an Ocean Optics tungsten-halogen light source (HL-2000-FHSA, emitting from $\sim 360-2400 \mathrm{~nm}$ ) and an Ocean Optics NIRQuest512 near-infrared spectrometer and associated laptop computer and OceanView software (covering the range $\sim 900$ to $1700 \mathrm{~nm}$ ). The spectrometer with a resolution of $\sim 3.1 \mathrm{~nm}$ FWHM uses a 512-element InGaAs array detector produced by Hamamatsu Photonics. The broad-band emission from the light source travels in a reflection probe that consists of 6 multimode illumination optical fibers around a center "read" optical fiber. The probe is positioned at 90-degrees from the MHCG test sample surface to measure the power reflectance of the undoped GaAs surface grating at near normal incidence. The reflection probe has a 25-degree full angle field of $\mathrm{v}$ iew. A polarizing filter is placed between the light source and the reflection probe (fiber bundle). An aluminum thin-film mirror standard on quartz is used as a reference to calibrate the test set-up. We measure a polished GaAs wafer piece as a reference with air as the incident medium and find the measured $R$ to be within 0.05 of the simulated $R$ from $900-1700 \mathrm{~nm}$ (i.e. falling within 0.32 to 0.30 ). Fig.5c illustrates the comparison of experimental and calculated power reflectance spectra in the range of the wavelengths from $900 \mathrm{~nm}$ to $1600 \mathrm{~nm}$. Our actual fabricated MHCG is not perfectly rectangular as designed and as simulated, but has slightly rounded bottom trench edges which alter (reduce) the power reflectance $(R)$ from the ideal $R$. To validate the our model we calculate $R$ for an MHCG with stripes of reconstructed shape based on the SEM image. The experimental power reflectance spectrum of power reflectance is very close to the calculated spectrum. The deviation may arise from different distance of reflected light to "read" optical fiber from reference mirror and grating, and also from possible imperfect periodicity of the grating parameters in our real-world MHCG structure.

In conclusion, we studied and elucidated important aspects of MHCG mirror designs which enable a power reflectance above 0.99 , which is necessary to support lasing in a VCSEL. We performed an exhaustive analysis of the geometrical parameters of MHCGs in a broad range of refractive indices from 1.75 to 4 . We defined LMPRs that relate to the various distributions of the optical field within the region of a given MHCG. We performed an analysis of the LMPR stopbands. In the case of LMPR 1 the $99 \%$ stopband was maximal and reached values above 0.06 for $n>3$ which is a typical value for AlGaAs/GaAs DBRs used in VCSELs.

\section{FUNDING INFORMATION}

This work is supported by the Polish National Science Center (project UMO-2014/15/B/ST7/05258). M. Gębski acknowledges the support of the Polish National Science Centre (project UMO2013/11/N/ST7/02826). J.A. Lott is supported by the German Research Foundation (Deutsche Forschungsgemeinschaft - DFG) via the Collaborative Research Center 787 (Sonderforschungsbereich 787) E. Haglund and A. Larsson are supported by the Swedish Foundation for Strategic Research.

\section{REFERENCES}

1. S.S. Lo, M.S. Wang and C.C. Chen, Optic Express 12(26), 6589-6593 (2004).

2. Y. Sakurai and F. Koyama, Jpn. J. Appl. Phys. 43(8B), 5828-5831 (2004).

3. S.S. Murtaza. K.A. Anselm, A. Srinivasan, B.G.Streetman, J.C. Campbell, J.C. Bean and L. Peticolas, Journal of Quantum Electronic, 31(10), 1819-1825 (1995).

4. R. Michalzik, VCSEL Fundamentals, Chap. 2 in "VCSELs - Fundamentals, Technology and Applications of Vertical-Cavity Surface-Emitting Lasers", Berlin, Germany, Springer-Verlag, 2013

5. H. Li, P. Wolf, P. Moser, G. Larisch, J.A. Lott and D. Bimberg, SPIE Newsroom, doi: 10.1117/2.1201411.005689

6. P. Moser J.A. Lott, G. Larisch and D. Bimberg, J. Lightw. Technol. 33(4), 825-831 (2015)

7. C.F.R. Mateus, M. C. Huang, Y. Deng, A.R. Neureuther, C.J. ChangHasnain, IEEE Photonics Technol. Lett., 16(2), 518-520 (2004).

8. C.F.R. Mateus, M. C. Huang, L. Chen, C.J. Chang-Hasnain and Y. Suzuki, IEEE Photon. Technol. Lett. 16(7), 1676-1678 (2004).

9. M.C.Y. Huang. Y. Zhou and C.J. Chang-Hasnain, Nat. Photonics 1(2), 119-122 (2007).

10. I.S. Chung. J. Mørk, P. Gilet and A. Chelnokov, IEEE Photon. Technol. Lett. 20(2), 105-107 (2008).

11. C. Sciancelepore, B.B. Bakir, X. Letarte, J.M. Fedeli, N. Olivier, D. Bordel, C. Seassal, P. Rojo-Romeo, P. Regreny and P. Viktorovitch, IEEE J. Lightwave Technology 29(6), 2015-2024 (2012).

12. S. Geoman, S. Boons, B. Dhoedt, K. Vandeputte, K. Caekebeke, P. Van Daele and R. Baets, IEEE Photon. Technol. Lett. 10(9), 1205-1207 (1998).

13. J. Lee, S. Ahn, H. Chang, J. Kim, Y. Park and H. Jeon, Opt. Express 17(25), 22535-22542 (2009).

14. A. Taghizadeh, G.C. Park, J. Mørk and I.S. Chung, Opt. Express, 22(18), pp. 21175-21184 (2014).

15. R. Magnusson, Opt. Letters 39(15), pp. 4337-4340 (2014).

16. M. Gębski, M. Dems, A. Szerling, M. Motyka, L. Marona, R. Kruszka, D. Urbańczyk, M. Walczakowski, N. Palıka, A. Wó jcik-Jedlińska, Q.J. Wang, D.H. Zhang, M. Bugajski, M. Wasiak and T. Czyszanowski, Opt. Express 23(9), 11674-11686 (2015).

17. M. Dems, R. Kotyński and K. Panajotov, Opt. Express, 13(9), 31963207 (2005).

18. C.J. Chang-Hasnain and W. Yang, Adv. Opt. Photonics 4(3), 379-440 (2012). 


\section{REFERENCES}

1. S.S. Lo, M.S. Wang and C.C. Chen, Semiconductor hollow optical waveguides formed by omni-directional reflectors, Optic Express 12(26), 6589-6593 (2004).

2. Y. Sakurai and F. Koyama, Control of Group Delay and Chromatic Dispersion in Tunable Hollow Waveguide with Highly Reflective Mirrors, Jpn. J. Appl. Phys. 43(8B), 5828-5831 (2004).

3. S.S. Murtaza. K.A. Anselm, A. Srinivasan, B.G.Streetman, J.C. Campbell, J.C. Bean and L. Peticolas, High-Reflectivity Bragg Mirrors for Optoelectronic Applications, Journal of Quantum Electronic, 31(10), 1819-1825 (1995).

4. R. Michalzik, VCSEL Fundamentals, Chap. 2 in "VCSELs - Fundamentals, Technology and Applications of Vertical-Cavity Surface-Emitting Lasers", Berlin, Germany, Springer-Verlag, 2013

5. H. Li, P. Wolf, P. Moser, G. Larisch, J.A. Lott and D. Bimberg, Verticalcavity surface-emitting lasers for optical interconnects, SPIE Newsroom, doi: $10.1117 / 2.1201411 .005689$

6. P. Moser J.A. Lott, G. Larisch and D. Bimberg, Impact of the Oxide-Aperture Diameter on the Energy-Efficiency, Bandwidth, and Temperature-Stability of 980-nm VCSELs, J. Lightw. Technol. 33(4), 825-831 (2015)

7. C.F.R. Mateus, M. C. Huang, Y. Deng, A.R. Neureuther, C.J. ChangHasnain, Ultra-broadband mirror using low index cladded subwavelength grating, IEEE Photonics Technol. Lett., 16(2), 518-520 (2004).

8. C.F.R. Mateus, M. C. Huang, L. Chen, C.J. Chang-Hasnain and Y. Suzuki, Broad-band mirror (1.12-1.62 $\mu \mathrm{m})$ using a subwavelength grating, IEEE Photon. Technol. Lett. 16(7), 1676-1678 (2004).

9. M.C.Y. Huang. Y. Zhou and C.J. Chang-Hasnain, A surface-emitting laser incorporating a high index-contrast subwavelength grating, Nat. Photonics 1(2), 119-122 (2007).

10. I.S. Chung. J. Mørk, P. Gilet and A. Chelnokov, Subwavelength GratingMirror VCSEL With a Thin Oxide Gap, IEEE Photon. Technol. Lett. 20(2), 105-107 (2008).

11. C. Sciancelepore, B.B. Bakir, X. Letarte, J.M. Fedeli, N. Olivier, D. Bordel, C. Seassal, P. Rojo-Romeo, P. Regreny and P. Viktorovitch, Quasi-3D Light Confinement in Double Photonic Crystal Reflectors VCSELs for CMOS-Compatible Integration, IEEE J. Lightwave Technology 29(6), 2015-2024 (2012).

12. S. Geoman, S. Boons, B. Dhoedt, K. Vandeputte, K. Caekebeke, P. Van Daele and R. Baets, First demonstration of highly reflective and highly polarization selective diffraction grating (GIRO-gratings) for longwavelenght VCSELs, IEEE Photon. Technol. Lett. 10(9), 1205-1207 (1998).

13. J. Lee, S. Ahn, H. Chang, J. Kim, Y. Park and H. Jeon, Polarizationdependent GaN surface grating reflector for short wavelength applications, Opt. Express 17(25), 22535-22542 (2009).

14. A. Taghizadeh, G.C. Park, J. Mørk and I.S. Chung, Hybrid grating reflector with high reflectivity and broad bandwidth, Opt. Express, 22(18), pp. 21175-21184 (2004).

15. R. Magnusson, Wideband reflectors with zero-contrast gratings, Opt. Letters, 39(15), pp. 4337-4340 (2014).

16. M. Gębski, M. Dems, A. Szerling, M. Motyka, L. Marona, R. Kruszka, D. Urbańczyk, M. Walczakowski, N. Pąlka, A. Wó jcik-Jedlińska, Q.J. Wang, D.H. Zhang, M. Bugajski, M. Wasiak and T. Czyszanowski, Monolithic high-index contrast grating: a material independent highreflectance VCSEL mirror, Opt. Express 23(9), 11674-11686 (2015).

17. M. Dems, R. Kotyński and K. Panajotov, Plane Wave Admittance Method - a novel approach for determining the electromagnetic modes in photonic structures, Opt. Express, 13(9), 3196-3207 (2005).

18. C.J. Chang-Hasnain and W. Yang, High-contrast gratings for integrated optoelectronics, Adv. Opt. Photonics 4(3), 379-440 (2012). 